\title{
Prevalence and Antibiotic Susceptibility Pattern of Streptococcus agalactiae Among Pregnant Women at Adigrat Zonal Hospital and Adigrat Health Center, Tigray, Ethiopia
}

\author{
Tsega Kahsay Gebremeskel ${ }^{1, ~ *, ~ T a m r a t ~ A b e b e ~ Z e l e k e ~}{ }^{2}$, Adane Mihret ${ }^{2}$, Mulugeta Desta Tikue ${ }^{3}$ \\ ${ }^{1}$ Adigrat University, College of Medicine and Health Sciences, Department of Bio Medical Science, Adigrat, Tigray, Ethiopia \\ ${ }^{2}$ Addis Abeba University, College of Health Sciences, Department of Microbiology, Immunology and Parasitology, Addis Abeba, Ethiopia \\ ${ }^{3}$ Adigrat Zonal Hospital, Gynecologist, Adigrat, Tigray, Ethiopia
}

\section{Email address:}

kahsaytsega@yahoo.com (T. K. Gebremeskel), tabebezeleke@gmail.com (T. A. Zeleke), adane-mihret@yahoo.com (A. Mihret), mulugeta-27@yahoo.com (M. D. Tikue)

\section{To cite this article:}

Tsega Kahsay Gebremeskel, Tamrat Abebe Zeleke, Adane Mihret, Mulugeta Desta Tikue. Prevalence and Antibiotic Susceptibility Pattern of Streptococcus agalactiae Among Pregnant Women at Adigrat Zonal Hospital and Adigrat Health Center, Tigray, Ethiopia. Journal of Gynecology and Obstetrics. Vol. 3, No. 2, 2015, pp. 29-35. doi: 10.11648/j.jgo.20150302.13

\begin{abstract}
Back ground: Streptococcus agalactiae or Lancefield group B Streptococci (GBS) is part of the microbiota of the mucous membranes of humans and animals, mainly colonizing the intestinal and genitourinary tracts. This study was undertaken to determine the carriage rate of $S$. agalactiae and to assess their antimicrobial susceptibility pattern. An attempt has been also made to identify the possible risk factors related with $S$. agalactiae colonization. Methods: Rectal and vaginal swabs were obtained from 150 pregnant women at 35-37 weeks of gestational period that attended antenatal clinic at Adigrat Zonal Hospital and Adigrat Health Center, Tigray, Ethiopia. Then the specimen was cultured on selective CHROMagarTMStrepB. In cases of positive cultures obtained, antibiotic susceptibility tests were carried out on all $S$. agalactiae isolates using the disc diffusion technique on Mueller-Hinton agar supplemented with $5 \%$ sheep blood. A univariate and multivariate binary logistic regression model was used to ascertain the association between the frequencies of colonization in relation to the different variables. Results: The prevalence of rectovaginal S. agalactiae colonization was (11.3\%). Bacterial resistance was detected to erythromycin (11.8\%) and clindamycin (17.6\%). By multi variant logistic regression analysis, prolonged rupture of membrane was associated with a higher colonization rate of $S$. agalactiae $(\mathrm{OR}=5.864,95 \% \mathrm{CI}=1.395-$ 24.643, P-value $=0.016$ ). Conclusion: The carriage rate of $S$. agalactiae among pregnant women in the present and a previous study in Gondar, remain low. The rates, risk factors of maternal GBS colonization may vary in different communities and need to be thoroughly evaluated in each country to allow the most appropriate preventive strategy to be selected.
\end{abstract}

Keywords: Prevalence, S. agalactiae, Pregnancy, Colonization, Ethiopia

\section{Background}

Streptococcus agalactiae or Lancefield group B Streptococci (GBS) is part of the microbiota of the mucous membranes of humans and animals, mainly colonizing the intestinal and genitourinary tracts [1]. In the 1970s, the bacterium emerged as the leading infectious cause of early neonatal morbidity and mortality in the United States. The great medical importance of this microorganism is causing of severe septicemia, pneumonia and meningitis of neonates $[2$, 3]. It is also associated with preterm labor or membrane rupture, as well as urinary tract infections, postpartum endometritis, postpartum wound infection, septic pelvic thrombophlebitis and endocarditis [4].

Results collected from different geographic regions indicated heterogeneous carriage rate of $S$. agalactiae in pregnant women [5-9]. A literature review study in SubSaharan Africa and in Middle East/North Africa indicates 19\% and $22 \%$, respectively, colonization rate of $S$. agalactiae [10]. The carriage rate of $S$. agalactiae in Gondar, Ethiopia is $9 \%$ in the mothers and 5\% in the neonates [11]. Puerperal infections and complicated abortion are some of the leading causes of 
maternal death in Ethiopia [12] which could be associated with S. agalactiae colonization.

S. agalactiae remain fully susceptible to penicillin $[13,14]$ as well as to most $\beta$-lactams, even though they have penicillin minimal inhibitory concentrations (MIC). However, recently identified very rare isolates with decreased susceptibility to penicillin have been reported in Japan [15] and USA [16]. Similarly, increased resistances to clindamycin and to erythromycin were reported in different part of the world [7, 17-22].

Therefore, as the $S$. agalactiae are worrying and continuing, the primary intent of this study was to determine the carriage rate of $S$. agalactiae among pregnant women and to determine the drug of choices to reduce transmission of $S$. agalactiae from colonized women to their neonates.

\section{Materials and Methods}

\subsection{Study Area}

The study was conducted at Adigrat Zonal Hospital and Adigrat Health Center which are located in Adigrat city, the administrative town of the Eastern Zone of Tigray region, Ethiopia. Adigrat town is about $891 \mathrm{kms}$ to North of the Ethiopian capital city Addis Abeba. The population of the town is about 130,000 . Adigrat Zonal Hospital is one of the 12 Hospitals in Tigray region. The two health institutions give health service like anti natal follow up and delivery.

\subsection{Study Design and Period}

A cross-sectional study was conducted at ANC clinic of Adigrat Zonal Hospital and Adigrat Health Center from the period of December 2011 to March 2012.

\subsection{Study Population}

The study populations were pregnant women who attended ANC follow up in Adigrat Zonal Hospital and Adigrat Health Center who were in their 35 to 37 weeks of gestational period.

Inclusion criteria: pregnant women voluntary to participate and whose gestation period is from 35 to 37 weeks.

Exclusion criteria: pregnant women who took antibiotics within one week before they came to the antenatal clinic service.

Sampling procedure: women were recruited consecutively as they came to ANC follow up.

\subsection{Sample Size Calculation}

The minimum sample size (n) was calculated by taking carriage rate of $S$. agalactiae in pregnant women from previous study done in Gondar university medical hospital and which was $9 \%$ carriage rate [11]. The margin of error (d) was 0.05 and the confidence interval $(\mathrm{Z} \alpha / 2)$ was $95 \%$.

$$
n=\frac{(Z \alpha / 2)^{2} *(p * q)}{d^{2}}=\frac{(1.96)^{2} *(0.09 * 0.91)}{(0.05)^{2}}=126
$$

Where; $\mathrm{n}=$ sample size; $\mathrm{z}=$ standard score corresponding to $95 \%$ confidence level $=1.96 ; \alpha=$ level of significance; $d=$ margin of error, $p=$ proportion and $q=1-p$.

Twenty percent (26) of the total calculated sample size $(n=126)$ was added for non response rate and the actual sample size was 152 .

\subsection{Statistical Analysis}

Data entry and analysis was done using Statistical Package for Social Science (SPSS) version 16 software. Descriptive summarization of data consisted of frequency counts and percentages were used to compare certain data. A univariate and multivariate binary logistic regression model was used to ascertain the association between the frequencies of colonization in relation to the different variables. All statistical tests were two tailed, and a $p$-value of $<0.05$ was considered as indicative of a statistically significant association.

\subsection{Data Collection}

After explaining the procedure and aim of the work, demographic data was collected using questionnaire by the trained personal.

\subsection{Sample Collection and Processing}

Using aseptic technique, rectal and vaginal swabs were collected as to the CDC recommendations [23]. For collection of vaginal and anal samples, first a swab from the mucosal secretions of the lower-third part of the vagina was obtained. Thereafter, the second swab was carefully inserted to the anal sphincter and gently rotated to touch the anal crypts. All swabs were collected by the clinical staff by the use of sterile double swabs and swabs were placed in Amies transport medium (Oxoid, England) and sent to the microbiology laboratory of Addis Pharmaceuticals Factory (APF). Specimens were processed in the laboratory within 24 hour of sample collection.

\subsection{Laboratory Procedures}

\subsubsection{Culture and Biochemical Test}

For isolation, Chromogenic medium (CHROM agar TM Strep B, France) was used and prepared following manufacturers instruction.

The two swabs were processed as one by direct inoculation on to CHRO Magar TM Strep B plate and incubated aerobically at $37^{\circ} \mathrm{C}$ for 24 hours. Suspected colonies of $S$. agalactiae i.e. mauve color (pink colonies) were confirmed by microscopy (gram positive cocci arranged in chains), catalase test (negative), CAMP test (positive) and serological test (presence of agglutination). If $S$. agalactiae is not identified after $24 \mathrm{~h}$, the media was re inoculated for additional $24 \mathrm{~h}$ to identify the suspected organism.

From CHROM agar TM Strep B, pink colonies were taken and sub cultured on to sheep blood agar plate (SBAP) then incubated under candle jar atmospheres for 18 hours. Finally colonies from SBAP were taken for serological test by latex 
agglutination method (PASTOREX ${ }^{\mathrm{TM}}$ Meningitis, France) according to the manufacturer's instruction.

\subsubsection{Antimicrobial Susceptibility Testing}

Antimicrobial susceptibility testing was carried out on isolates $S$. agalactiae using disc diffusion technique on Mueller-Hinton agar (Oxoid, England) supplemented with 5\% sheep blood. A suspension of the test organism was prepared by removing 3-5 colonies from a pure culture plate by emulsifying in $3 \mathrm{ml}$ of sterile physiological saline and incubated at $37^{\circ} \mathrm{C}$ until the turbidity of the suspension become matched with turbidity standard equivalent to 0.5 McFarland. Using a sterile swab, the surface of MuellerHinton agar was completely covered by pressing and rotating the swab against the side of the tube above the level of suspension. After the plate had dried (3-5 minutes), using sterile forceps the six discs were evenly distributed on the inoculated plate and incubated at $37^{\circ} \mathrm{c}$ in $5 \% \mathrm{CO}_{2}$ for 24 hours according to the guideline recommendations of $\mathrm{CDC}$ [23]. Using a ruler, zone of inhibition was measured and interpreted according to Clinical and Laboratory Standards Institute (CLSI) guide lines as 'Resistant", 'Intermediate/Moderately susceptible', 'Susceptible' [24].

The following antimicrobial discs and concentrations (in brackets) were used: ampicillin $(10 \mu \mathrm{g})$; amoxicillin $(2 \mu \mathrm{g})$; erythromycin $(15 \mu \mathrm{g})$; clindamycin $(2 \mu \mathrm{g})$, Vancomycin $(30$ $\mathrm{mg}$ ), and penicillin $\mathrm{G}(10 \mathrm{mg}$ ) (All of the antibiotics are product of Oxoid, England).

\subsubsection{Quality Control}

Staphylococcus aureus (ATCC-25923) was used as a quality control throughout the study for culture, antimicrobial susceptibility testing and CAMP test.

Enterococcus fecalis (ATCC -25212) was used as negative control for CAMP testing. The control strains were obtained from Ethiopian Health and Nutrition Research Institute (EHNRI).

Sample was collected and processed aseptically by using standard operating procedure (SOP).

\subsection{Ethical Considerations}

The study was part of an M.Sc research project. So it was ethically approved by the Department of Microbiology, Immunology and Parasitology Ethical Review Committee (DERC) of Addis Ababa University. Written informed consent was obtained from all the study participants. In the event of a positive culture for $S$. agalactiae, the result was communicated with the gynecologist for appropriate management.

\section{Results}

\subsection{Socio-Demographic Characteristics of Study Participants}

One hundred fifty pregnant women were included in the study with $98.7 \%$ response rate. A socio-demographic characteristic of study participants was summarized in Table 1. The mean age of the women in this study was $26.14 \pm 4.9$ year (ranging from 14 to 40 years and its median was 25 years). Many of the pregnant women, 109(72.7\%) were in 21-30 years age group followed by greater than or equal to 31 years $24(16 \%)$ and less than or equal to 20 years $17(11.3 \%)$. The mean gestational age $( \pm \mathrm{SD})$ at examination was $35.7 \pm 0.77$ weeks (median 35.5 weeks, range 35-37 weeks). Half of the study participants $(50 \%)$ were in 35 weeks of gestational age, $47(31.3 \%)$ of them were in 36 weeks of gestational age and $28(18.7 \%)$ were in 37 weeks of gestational age.

Table 1. Socio-demographic characteristics of study participants investigated for S. agalactiae colonization at Adigrat Zonal Hospital and Adigrat Health Center, Tigray, Ethiopia from [December 2011-March 2012].

\begin{tabular}{lll}
\hline Variables & No Study participants $(\mathbf{N}=\mathbf{1 5 0})$ & \% of Study participants \\
\hline Age, yrs & & \\
$\leq 20$ & 17 & 11.3 \\
$21-30$ & 109 & 72.7 \\
$\geq 31$ & 24 & 16 \\
Gravida & 110 & 73.3 \\
Primigravida $\leq 3$ & 40 & 26.7 \\
Multi gravida $>3$ & & \\
Gestational age in wks & 75 & 50 \\
35 & 47 & 31.3 \\
36 & 28 & 18.7 \\
37 & & \\
HIV & 3 & 2 \\
Yes & 147 & 98 \\
No & & \\
\hline
\end{tabular}

\subsection{Carriage Rate of $S$. agalactiae}

As shown in Table 2 among the 150 pregnant women evaluated $93(62 \%)$ of them were from health center and $57(38 \%)$ were from hospital. Seventeen $(11.3 \%)$ of the pregnant women were colonized by $S$. agalactiae. Four (7\%) and $13(14 \%)$ of the pregnant women were those who have attended ANC follow up at Adigrat Zonal Hospital and Adigrat Health Center, respectively. Thirteen (76.5\%) of the isolates were from health center and $4(23.5 \%)$ were from 
hospital.

Table 2. Age distribution of S. agalactiae colonized pregnant women at Adigrat Zonal Hospital and Adigrat Health Center, Tigray, Ethiopia from [December 2011-March 2012].

\begin{tabular}{llll}
\hline Age in years & Hospital $(\mathbf{n}=\mathbf{5 7})$ No. $(\%)$ & Health Center $(\mathbf{n}=\mathbf{9 3})$ No. $(\%)$ & Total $\boldsymbol{S}$. agalactiae isolated $(\mathbf{n}=\mathbf{1 7})$ No. $(\%)$ \\
\hline$\leq 20$ & $0(0)$ & $1(1.07)$ & $1(5.9)$ \\
$21-30$ & $3(5.26)$ & $10(10.75)$ & $13(76.5)$ \\
$\geq 31$ & $1(1.75)$ & $2(2.15)$ & $3(17.6)$ \\
Total & $4(7.01)$ & $13(13.97)$ & $17(100)$ \\
\hline
\end{tabular}

\subsection{Risk Factors Associated with Colonization of S. agalactiae}

After analyzing the carriage rate of $S$. agalactiae, next we assessed the risk factors associated with colonization of $S$. agalactiae in the pregnant women. Among the several factors assessed on univariate analysis (initial analysis), there was statistically significant association in the isolation frequency of $S$. agalactiae with Preterm labor $(\mathrm{OR}=0.115,95 \% \mathrm{CI}=$ 0.015-0.873, P-value $=0.037)$ and $\mathrm{PROM}(\mathrm{OR}=0.154,95 \%$ $\mathrm{CI}=0.38-0.615, \mathrm{P}$-value $=0.008)$. However none of the other risk factors such as age, early neonatal death, gestational age and other history of the study participants had not any significant association in the isolation frequency of $S$. agalactiae $(\mathrm{p}>0.05)$ as provided in Table 3 .

Finally, after univariate analysis was done we did multivariate logistic analysis, in order to ascertain association by adjusting for confounders. For the 2 risk factors (Preterm labor and PROM) in which statistically significant association was obtained by univariate analysis, stepwise logistical regression identified PROM only had significant association with $S$. agalactiae colonization $(\mathrm{OR}=5.864,95 \%$ $\mathrm{CI}=1.395-24.643$, P-value=0.016). Prolonged rupture of membrane independently contributes 5.86 times for colonization of $S$. agalactiae as provided in Table 4.

Table 3. Univariate analysis of respondents' characteristics related with S. agalactiae colonization at Adigrat Zonal Hospital and Adigrat Health center, Tigray, Ethiopia from [December 2011-March 2012].

\begin{tabular}{|c|c|c|c|c|c|c|}
\hline Variable & Total & Yes & No & $95 \%$ CI & OR & P-value \\
\hline \multicolumn{7}{|l|}{ Preterm labor } \\
\hline Yes & 4 & 2 & 2 & $0.015-0.873$ & 0.115 & 0.037 \\
\hline No & 146 & 15 & 131 & & 1 & \\
\hline \multicolumn{7}{|l|}{ Parity } \\
\hline Primi parity $(\leq 3)$ & 130 & 14 & 116 & $0.178-2.630$ & 0.684 & 0.580 \\
\hline Multi parity $(>3)$ & 20 & 3 & 17 & & 1 & \\
\hline \multicolumn{7}{|l|}{ PROM } \\
\hline Yes & 10 & 4 & 6 & $0.38-0.615$ & 0.154 & 0.008 \\
\hline No & 140 & 13 & 127 & & 1 & \\
\hline \multicolumn{7}{|l|}{ Gestational age } \\
\hline 35 & 75 & 8 & 67 & & 1 & \\
\hline 36 & 47 & 5 & 42 & $0.385-5.059$ & 1.396 & 0.612 \\
\hline 37 & 28 & 4 & 24 & $0.343-5.718$ & 1.400 & 0.639 \\
\hline
\end{tabular}

CI-Confidence Interval; OR-Odds Ratio; 1-Reference Group and PROM-Prolonged Rupture of Membrane

Table 4. Multivariate analysis of respondents' characteristics related with $S$. agalactiae colonization at Adigrat Zonal Hospital and Adigrat Health center, Tigray, Ethiopia from [December 2011-March 2012].

\begin{tabular}{llll}
\hline Variable & $\mathbf{9 5} \% \mathbf{C I}$ & OR & P=value \\
\hline $\begin{array}{l}\text { Preterm labor } \\
\text { Yes }\end{array}$ & & 1 & \\
No & $0.822-61.438$ & 7.105 & 0.075 \\
PROM & & & \\
Yes & $1.395-24.643$ & 5.864 & 0.016 \\
No & & 1 & \\
\hline
\end{tabular}

CI-Confidence Interval; OR-Odds Ratio; 1-Reference Group

\subsection{Antibiotic Susceptibility Pattern of Isolates}

As shown in Table 5 all the 17 isolates were susceptible to ampiciillin (100\%), penicillin G (100\%), vancomycin (100\%), and amoxacillin (100\%). However, 2 isolates were resistant to erythromycin $(11.8 \%)$ and three isolates to clindamycin (17.6\%). Common resistant to erythromycin and clindamycin was found in two isolates. Furthermore, intermediate susceptibility was also detected against erythromycin and clindamycin in 2 isolates (11.8\%) for each. 
Table 5. Antibiotic susceptibility profiles of S. agalactiae isolated from pregnant women in Adigrat Zonal Hospital and Adigrat Health Center, Tigray, Ethiopia from [December 2011-March 2012].

\begin{tabular}{llll}
\hline Antibiotic & & \multicolumn{2}{l}{ No. (\%) of the isolates with indicated Antibiotic response } \\
\hline Susceptible & & Intermediate & Resistant \\
\hline Penicillin $\mathrm{G}^{\mathrm{a}}$ & $17(100)$ & ---- & ---- \\
Amoxacillin $^{\mathrm{a}}$ & $17(100)$ & ---- & --- \\
Ampicillin $^{\mathrm{a}}$ & $17(100)$ & --- & - \\
Clindamycin $^{\mathrm{a}}$ & $12(70.6)$ & $2(11.8)$ & $3(17.6)$ \\
Erythromycin $^{\mathrm{a}}$ & $13(76.5)$ & $2(11.8)$ & $2(11.8)$ \\
Vancomycin $^{\mathrm{a}}$ & $17(100)$ & ---- & ---- \\
\hline
\end{tabular}

${ }^{\mathrm{a}}=$ Clinical and Laboratory Standards Institute (CLSI) breakpoint for Streptococcus spp $\beta$ - haemolytic group [24]

\section{Discussion}

This study showed that $11.3 \%$ of pregnant women who attended ANC follow up in the study area had rectovaginal colonization with $S$. agalactiae. A comparable rate of colonization with the current study was reported in the previous study done in Gondar, Ethiopia (9\%) [11], and in Saudi Arabia (9.2\%) [25]. This prevalence is within the often quoted range of between $5.2 \%-34.3 \%$ in different parts of the world $[5,6,9,17,26,27]$. This highlights the importance of individualizing preventive strategies according to local colonization rates.

In our study, all $S$. agalactiae isolates were uniformly sensitive to penicillin, which is in agreement with previous reports [7, 17-21]. From this we recommend penicillin $G$ as the best antibiotic for the treatment of $S$. agalactiae.

Since the introduction of protocols for the prevention of the newborn $S$. agalactiae diseases, the use of erythromycin and clindamycin has increased, especially in patients allergic to penicillin [23]. At the same time, there was an increase in rates of $S$. agalactiae resistance to these antibiotics, according to the results observed in studies performed in different countries [17-19].

In the current study, the prevalence of resistance to clindamycin was $17.6 \%$. This was in agreement with finding observed in Egypt (17.9\%) [17], Tanzania (17.6\%) [18] and France $(17.5 \%)$ [19], but the rate was higher than that of other reports from Thammasat (3\%) [7], Czech Republic (3.2\%) [21], East Lansing (6.4\%) [22].

In addition, the resistance to erythromycin was $11.8 \%$ this was in agreement with resistance observed in Argentina (9.7\%) [20], and in Canada (14\%) [28]. Therefore, we recommend the susceptibility testing of GBS colonizing mothers during pregnancy.

Data collected from questionnaires showed $S$. agalactiae colonization among pregnant women was significantly correlated with the PROM $(\mathrm{OR}=5.864,95 \% \mathrm{CI}=1.395$ 24.643, $\mathrm{P}$-value $=0.016$ ). This was in agreement with a recent study in India [29] and in Iran [26]. But this is in contrast with other study in Tanzania [18]. However no significant relationship was found between maternal $S$. agalactiae carriage rate and number of parity and abortion history $(p>0.05)$. This might be probably due to smaller sample size, geographical or socio economic characteristics of the study participants. This was in agreement with previous findings in
Iran [26]. But other colleagues get association; pregnant women with previous spontaneous abortion were more frequently colonized by $S$. agalactiae [31]. In the current study colonization was not significantly greater in multigravid $(>3)$ than in primigravid $(\leq 3)$ women and this is in agreement with other collogues in India [29], in Trindad [32] and in Peru [27]. But this is in contrast with a study in south India [31].

Perinatal $S$. agalactiae disease is both an expensive and serious condition that can be effectively prevented by relatively low-cost and fast screening strategies during gestation. The carriage rate of $S$. agalactiae in the present study was similar with industrialized countries carriage rate and prevention method of $S$. agalactiae is necessary in Adigrat. Maternal and child health are high priorities for international development. Currently, Ethiopia is on the way of development and works to decrease maternal and neonatal, mortality and morbidity. In the current study we administered to the colonized pregnant women penicillin $\mathrm{G}$ according to the recommendation by CDC [23] to reduce the transmission of the bacteria from mother to the neonate and we expect our study may contribute its own merit to achieve millennium developmental goal of Ethiopia.

Neonates born from the study participants were not included in the study due to time and budget constraints. In addition, it was not possible to do sero type of the isolates due to difficulty to get the reagent. These are considered as limitation of the study. Beside this further study must be under taken to know the transmission rate to their neonates and to know the sero type distribution of S.agalactia in the study area.

\section{Conclusion}

Our results clearly indicate that rates of GBS colonization among pregnant women have reached comparable to this previously done in Gondar, Ethiopia. Ampicillin, penicillin G, vancomycin and amoxicillin were found to be effective drugs to isolates of $S$. agalactiae. However, resistant isolates were found to commonly prescribed antibiotics such as erythromycin and clindamycin. Study participants with history of PROM were more colonized than without PROM. The rates, risk factors of maternal GBS colonization may vary in different communities and need to be thoroughly evaluated in each country to allow the most appropriate preventive strategy to be selected. 


\section{Authors' Contributions}

TK was the primary researcher, conceived the study, designed, participated in data collection, conducted data analysis and drafted the manuscript for publication. TA, AM and MD assisted in data collection and reviewed the initial drafts of the manuscript. All authors read and approved the final manuscript.

\section{Acknowledgements}

We acknowledge all the staff members of Adigrat zonal hospital, Adigrat health center \& Addis Pharmaceutical factory for their technical support during data collection and processing.

We would like also to extend our best gratitude to Alberto Lerner, General Manager of the CHROMagar Company the only supplier of CHROM agar ${ }^{\mathrm{TM}}$ Streptococci medium for the generous gift of the medium which was the most important and mandatory material used for this research project.

\section{References}

[1] Centers for Disease Control: Prevention of perinatal group B Streptococcal disease: a public health perspective. MMWR Recomm Rep 1996, 45(RR-7):1-24.

[2] Schuchat A: Prevention effectiveness case study: institutionalizing prevention of group B Streptococcal infections national center for infectious diseases centers for disease control and prevention Atlanta, Georgia 1995, 227-32.

[3] Rollins DM: Streptococcus summary. [http://life.umd.edu/classroom/bsci424].

[4] Murray PR, Rosenthal KS and Pfaller MA: Medical microbiology. (5thed) USA: 2005: 247-250.

[5] Bergseng H, Bevanger L, Rygg M, Bergh K: Real-time PCR targeting the sip gene for detection of group B Streptococcus colonization in pregnant women at delivery. J Med Microbiol 2007, 56(pt 2):223-8.

[6] Knox JM: Group B Streptococcal infection: a review and update. Br J Vener Dis 1979, 55(2):118-20.

[7] Tor-Udom S, Tor-Udom P, Hiriote W: The prevalence of Streptococcus agalactiae (Group B) colonization in pregnant women at Thammasat Hospital. J Med Assoc Thai 2006, 89(4):411-4.

[8] Chua S, Arulkumaran S, Chow C, Kumarasinghe G, Selamat N, Kuah BG, Ratnam SS: Genital group B Streptococcus carriage in the antenatal period: its role in PROM and preterm labor. Singapore MED J 1995, 36(4):383-5.

[9] Church DL, Baxter H, Lioyd T, Miller B, Elsayed S: Evaluation of StrepB carrot broth versus lim broth for detection of group B Streptococcus colonization status of near-term pregnant women. J Clin Microbiol 2008, 46(8):2780-2.

[10] Stoll BJ, Schuchat A: Maternal carriage of group B Streptococci in developing countries. Pediatr Infect Dis J 1998, 17(6):499-503.
[11] Schmidt J, Halle E, Halle H, Mohammed T, Gunther E: Colonization of pregnant women and their newborn infants with group B Streptococci in the Gondar College of medical sciences. Ethiop Med J 1989, 27(3):115-9.

[12] World Health Organization: Country Cooperation Strategy 2008-2011.

[13] Murayama SY, Seki C, Sakata H, Sunaoshi K, Nakayama E, Iwata S, Sunakawa K, Ubukata K; Invasive Streptococcal Disease Working Group. Capsular Type and Antibiotic Resistance in Streptococcus agalactiae Isolates from Patients, Ranging from Newborns to the Elderly, with Invasive Infections. Antimicrobial Agents and Chemotherapy 2009, 53(6): 2650-2653.

[14] Orrett FA: Colonization with Group B streptococci in pregnancy and outcome of infected neonates in Trinidad. Pediatr Int 2003, 45:319-323.

[15] Kouji K, Noriyuki N, Yukiko N, Jun-ichi W, Satowa S, Keigo S, Yoshichika A: Predominance of sequence type 1 group with serotype VI among group B streptococci with reduced penicillin susceptibility. J Antimicrob Chemother 2011, 66(11): 2460-2464.

[16] Noriyuki N, Kouji K, Yukiko N, Hiroaki Y, Yoshichika A. Molecular characterization of group B streptococci with reduced penicillin susceptibility recurrently isolated from a sacral decubitus ulcer. J Antimicrob Chemother 2009, 64(6):1326-1328.

[17] Abdelmoaty T, Wafaa Z, Kawthar M: Prevalence and antibiotic susceptibility of anogenital group B streptococci colonization in pregnant women. Egypt J Med Lab 2009, 18(2):105-111.

[18] Joachim A, Matee MI, Massawe FA, Lyamuya EF: Maternal and neonatal colonization of group B Streptococcus at Muhimbili national hospital in Dares Salaam, Tanzania: prevalence, risk factors and antimicrobial resistance. BMC Public Health 2009, 9:437.

[19] De Mouy D, Cavallo JD, Leclercq R, Fabre R: Antibiotic susceptibility and mechanisms of erythromycin resistance in clinical isolates of Streptococcus agalactiae. Antimicrob Agents Chemother 2001, 45(8):2400-2.

[20] Quiroga M, Pegels E, Oviedo P, Pereyra E, Vergara M: Antibiotic susceptibility patterns and Prevalence of Group B Streptococcus Isolated from Pregnant women in Misiones, Argentina. Brazilian Journal of Microbiology 2008, 39: 245250 .

[21] Motlova J, Strakova L, Urbaskova P, Sak P, Sever T: Vaginal and rectal carriage of Streptococcus agalactiae in the Czech Republic: incidence, serotypes distribution and susceptibility to antibiotics. Indian J Med Res 2004, 119 (Suppl):84-7.

[22] Manning SD, Schaeffer KE, Springman AC, Lehotzky E, Lewis MA, Ouellette LM, Wu G, Moorer GM, Whittam TS, Davies HD: Genetic diversity and antimicrobial resistance in group B Streptococcus colonizing young, non-pregnant women. Clin Infect Dis 2008, 47(3):388-90.

[23] Centers for Disease Control and Prevention: Prevention of perinatal group B Streptococcal disease revised guidelines from CDC, 2010. MMWR Recomm Rep 2010, 59 (RR-10):136. 
[24] Clinical and Laboratory Standards Institute: Performance standards for antimicrobial susceptibility testing; Twentieth informational supplement.M100-S20 2010, 30(1).

[25] Uduman SA, ChatterjeeTK, Al-Mouzan MI, AL-Suleiman S: Group B Streptococci colonization among Saudi women in labor and neonatal acquisition. Int J Gynaecol Obstet 1985, 23(1):21-4.

[26] Nahaei MR, Ghandchilar N, Bilan N, Ghahramani P: Maternal carriage and neonatal colonization of Streptococcus agalactiae in Tabriz, Northwest Iran. Iran J Med Sci2007, 32(3):177-81.

[27] Collins TS, Calderon M, Gilman RH, Vivar A, Charache P: Group B Streptococcal colonization in a developing country: it's association with sexually transmitted disease and socioeconomic factors. Am J Trop Med Hyg 1998, 59(4):6336.
[28] Andrews JI, Diekema DJ, Hunter SK, Rhomberg PR, Pfaller MA, Jones RN, Doern GV: Group B Streptococcicausing neonatal bloodstream infection: antimicrobial susceptibility and serotyping results from sentry centers in the western hemisphere. Am J Obstet Gynecol 2000, 183(4):859-62.

[29] Dechen TC, Sumit K, Ranabir P: Correlates of vaginal colonization with group B Streptococci among pregnant women. J Glob Infect Dis 2010, 2(3):236-41.

[30] Sharmila V, Joseph NM, ArunBabu T, Chaturvedula L, Sistla S: Genital tract group B Streptococcal colonization in pregnant women: a South Indian perspective. J Infect DevCtries 2011, 5(8):592-5.

[31] Orrett FA, Olagundoye V: Prevalence of group B Streptococcal colonization in pregnant third trimester women in Trinidad. J Hosp Infect 1994, 27(1):43-8. 